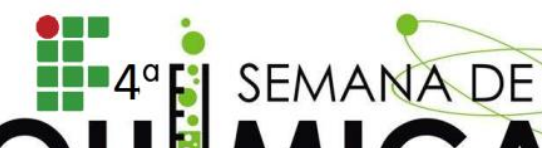 \\ QU MICA
}

\section{PROBLEMÁTICA DO USO DE AGROTÓXICOS NO RIO GRANDE DO NORTE}

NETO, N.R.S*.(IFRN); LEITE, A.D.(IFRN); SOUSA, E.E.(IFRN); SILVA, W.L.A.(IFRN)

\section{RESUMO}

Este trabalho tem como objetivo de informar o uso de agrotóxicos e noções de perigo, contaminação ambiental por agrotóxicos e agrotóxicos mais utilizados no Rio grande do norte. como adotar medidas para diminuir o uso de agrotóxico, aliadas à falta de assistência técnica especializada, gera além de efeitos nocivos à saúde, uma tendência ao descuido em relação à contaminação ambiental. 0 desenvolvimento da agricultura sustentável deve ser estimulado, pois contribuirá para a conservação do ambiente e a minimização dos efeitos negativos desses compostos na saúde humana e ambiental.

Estudar esta temática configura ferramenta importante visando propor políticas e programas de educação sanitária voltada a orientar e preparar a classe produtiva quanto aos procedimentos corretos para uso destes insumos, considerados atualmente como importantes instrumentos de auxílio na produção agrícola, mas que carecem de melhor conscientização e controle quanto ao seu uso.

Buscou-se nesta pesquisa mostrar como o Rio grande do Norte e seus municípios estão sendo envolvidos com as práticas inadequadas quanto ao uso excessivo de agrotóxicos, uma vez que estes podem trazer sérios danos ao homem e ao meio ambiente, tais como riscos de contaminações de pessoas e animais, dos alimentos produzidos, dos mananciais e do meio ambiente como um todo.

PALAVRAS-CHAVE: Contaminação, Riscos, Saúde

PROBLEMATIC PESTICIDE USE OF RIO GRANDE DO NORTE

\section{ABSTRACT}

This paper aims to inform the use of pesticides and dangerous notions of environmental contamination by

pesticides and pesticides commonly used in large northern Rio. how to adopt measures to reduce the use of pesticides, coupled with the lack of technical 
expertise, generates besides harmful health effects, a tendency to carelessness in relation to environmental contamination. The development of sustainable agriculture should be encouraged because it will contribute to the conservation of the environment and minimizing the negative effects of these compounds on human and environmental health.

Studying this issue sets important tool aimed at proposing policies and health education programs aimed to guide and prepare the productive class and the correct procedures for use of these inputs, currently considered as important aid instruments in agricultural production, but they need better awareness and control as to its use.

we sought in this research show how the great River of the North and its municipalities are involved with inappropriate practices for excessive use of pesticides, as these can cause serious damage to man and the environment, such as contamination risk people and animals, produced food, water sources and the environment as a whole.

KEY-WORDS: contamination, scratchs, cheers 


\section{PROBLEMÁTICA DO USO DE AGROTÓXICOS NO RIO GRANDE DO NORTE}

\section{INTRODUÇÃO}

O Rio Grande do Norte figura atualmente é um dos estados produtores de alimentos e principalmente certos vegetais no Brasil, dentre elas o intensivo uso de agrotóxicos, que contribue de forma determinante para o alto índice de produtividade das mais diversas atividades agrícolas e de cultivo.

Diante desta realidade, para a realização deste estudo, destacamos os seguintes fatores relevantes: O Rio Grande Do Norte figura entre os estados com maiores volumes de vendas de agrotóxicos; casos de intoxicações têm sido cada vez mais relatados no estado, principalmente entre as pessoas que os manipulam; a presença de resíduos de agrotóxicos na água, nos solos e principalmente em alimentos são constantemente divulgados em várias instituições. Dentre estes estudos se destaca o Programa de Análise de Resíduos em Alimentos, desenvolvido pela Agência de Vigilância Sanitária (ANVISA) onde vários estados da federação são participantes, inclusive o Rio Grande do Norte.

Diante do quadro que envolve desde a produção do agrotóxico, transporte, sua comercialização até propriamente o uso inadequado quando aplicado em excesso e muitas vezes de forma desnecessária, e considerando os elevados e graves índices de contaminações ambientais, em alimentos e de pessoas, o trabalho mostra uma importante ferramenta para discussão, realizando uma análise para se vislumbrar estratégias de enfrentamento visando minimizar os problemas decorrentes da presença dos grandes volumes de agrotóxicos despejados no meio ambiente ao longo dos últimos anos.

Observa-se que há um direcionamento ao cumprimento das exigências legais e aos requisitos adequados de segurança, quando do uso dos agrotóxicos. Farse-á uma análise na interferência que a aplicação de um processo educativo, bem como uma fiscalização efetiva junto aos produtores resistentes causaria de impacto positivo para a diminuição da incidência de casos de intoxicações, contaminação ambiental e dos alimentos por eles produzidos em decorrência do mau uso dos agrotóxicos. 


\section{MATERIAIS E MÉTODOS}

Rio Grande do Norte é uma das 27 unidades federativas do Brasil é dividido em 167 municípios e sua área total é de $52.811,126 \mathrm{~km}^{2}$. Com mais de 3,4 milhões de habitantes, o Rio Grande do Norte é o décimo sexto estado mais populoso do Brasil, o qual contabiliza 245 mil agricultores (wikipedia.org-pág-1).

A agricultura e a agropecuária são importantes atividades econômicas, sendo a agricultura familiar de subsistência um destaque na modalidade de cultivo, ainda que prejudicada pelas constantes estiagens.

A pesquisa foi direcionada para a coleta de informações específicas ao tema relevante, ou seja, relaciona à forma pela qual o produtor rural adquire e utiliza os agrotóxicos no desenvolvimento das atividades rurais e também o levantamento do uso graduado desse produto na região.

Com isso, a obtenção dos agrotóxicos se faz com muita facilidade e pouco se sabe sobre o uso, devido esse material que é altamente prejudicial ao meio ambiente e também ao homem.

A tabela mostra os principais agrotóxicos utilizados e as suas quantidades específicas, bem como totais de campos analisadosos :

\begin{tabular}{|c|c|c|}
\hline AGROTÓXICOS APURADOS & $\begin{array}{l}\text { QUANTIDADE DE } \\
\text { AGROTÓXICOS APURADOS } \\
\text { POR UNIDADE }\end{array}$ & $\begin{array}{l}\text { TOTALDE CAMPOS } \\
\text { ANALISADOS }\end{array}$ \\
\hline PIRATE & 6 \\
\hline LANNATEBR & 6 \\
\hline ACTARA & 2 \\
\hline SPORTAK & 1 \\
\hline CYPTRIN & 1 \\
\hline TRIGARD 75O & 2 \\
\hline EVIDENCE & 4 \\
\hline RIDOMIL & 1 \\
\hline KASUMI & 1 \\
\hline SAURUS & 2 \\
\hline DITHANE & 2 \\
\hline FASTAC 100 & 1 \\
\hline FEGATEX & 2 \\
\hline PREMIO & 2 \\
\hline VERTIMEC & 5 \\
\hline KATARE & 3 \\
\hline DACOBREWP & 1 \\
\hline CONFIDOR & 3 \\
\hline POLYTRIN & 2 \\
\hline RECOP & 1 \\
\hline
\end{tabular}


A exposição de forma crônica (exposição a baixas doses por longos períodos) pode desencadear o desenvolvimento de doenças tanto em trabalhadores quanto na população exposta a estes compostos, seja no ambiente, ou com a alimentação. Em geral, os riscos para a saúde humana decorrentes da exposição a agrotóxicos são, o desenvolvimento de câncer, mau formação e danos para o sistema nervoso e funcionamento do sistema endócrino (RANGEL; ROSA; SARCINELLI, 2011, p 436).

De acordo com Lyznicki et. al., (1997), citado por Soares (2010, p. 17), os efeitos dos agrotóxicos na saúde humana, especialmente os crônicos, não têm sido caracterizados adequadamente, pois os efeitos tardios de alguns desses químicos podem se tornar aparentes após anos de exposição.

Ao serem caracterizados adequadamente, pois os efeitos tardios de alguns desses produtos químicos podem se tornar aparentes após anos de exposição.

Resultados do Programa de Análise de Resíduos de Agrotóxicos (PARA), da (ANVISA), que avaliou os níveis de agrotóxicos nos alimentos de origem vegetal, identificou a existência de amostras insatisfatórias, em aproximadamente 31\% em 2011 e $15 \%$ em 2012.

Considerando para busca o termo "praguicidas" como contaminante potencial (informação registrada nas fichas de campo das áreas cadastradas no SISSOLO), foram encontradas 354 áreas no Rio Grande do Norte, nos seguintes municípios: Açu, Canguaretama, Ceará Mirim, Macaíba, Mossoró, Natal, Parnamirim e São Gonçalo do Amarante. Nessas áreas, estima-se que a população exposta seja de 922.583 pessoas. Desse conjunto, 41 áreas são agrícolas, localizadas nos municípios de Mossoró, Natal e São Gonçalo do Amarante, e, em todas, foi registrado o uso de agrotóxicos. Usando como critério a classificação de áreas "depósitos de agrotóxicos", a busca no SISSOLO resultou em 5 áreas cadastradas em Canguaretama e 1 em Natal (Base de UBV do estado), nas quais a estimativa da população exposta é de 3.500 pessoas. Embora a população exposta tenha sido apenas estimada, os registros no SISSOLO indicam que a exposição a agrotóxicos é um 
importante problema de saúde pública no Rio Grande do Norte (RIO GRANDE DO NORTE, 2013, p. 17).

\section{Incidência de Intoxicações por Agrotóxicos}

Segundo a Portaria no 1.271, de 6 de junho de 2014 (revoga Portaria no 104, de 25 de janeiro de 2011), a intoxicação por agrotóxicos faz parte da Lista de Notificação Compulsória (LNC) e deve ser notificada através da ficha de intoxicações exógenas do Sistema de Informação de Agravos de Notificação (SINAN).

Segundo SINAN, No Rio Grande do Norte a análise conjunta da evolução da área de produção agrícola, do consumo de agrotóxicos e da incidência das intoxicações, no mesmo período, apesar das oscilações, revelaram aumento na taxa de consumo de agrotóxicos, apesar da redução na área de plantio. Além disso, a incidência de intoxicações acompanha a tendência de aumento de consumo de agrotóxicos, variando de 0,10 para 0,77 casos por 100.000 habitantes, apresentando um aumento de registro em 2011.

Baseando-se nos registros os munícipios com maiores usos abusivos de agrotóxicos representados na tabela abaixo. 
Tabela 2: Municipios do Rio Grande do Norte que utilizam maior número de tipos de agrotóxicos.

MUNICÍPIO

NÚMERO DE AGROTÓXICOS

UTILIZADOS

\begin{tabular}{|l|l|}
\hline BARAÚNA & 191 \\
\hline MOSSORÓ & 168 \\
\hline NATAL & 115 \\
\hline SÃO JOSÉ DE MIPIBU & 91 \\
\hline JANDAIRA & 81 \\
\hline TOUROS & 76 \\
\hline CEARÁ MIRIM & 73 \\
\hline ALTO DO RODRIGUES & 70 \\
\hline APODI & 67 \\
\hline IPANGUAÇU & 65 \\
\hline PUREZA & 65 \\
\hline UPANEMA & 64 \\
\hline MACAIBAA & 62 \\
\hline AÇU & 60 \\
\hline VERA CRUZ & 59 \\
\hline CURRAIS NOVOS & 56 \\
\hline NÍSIA FLORESTA & 55 \\
\hline RIO DO FOGO & 52 \\
\hline ALMINO AFONSO & 44 \\
\hline GOVERNADOR DIX- SEPT ROSADO & 44 \\
\hline EXTREMOZ & 39 \\
\hline PARNAMIRIM & 39 \\
\hline PARELHAS & 34 \\
\hline SÃO GONÇALO DO AMARANTE & 34 \\
\hline ACARI & 32 \\
\hline
\end{tabular}

Fonte: IDIARN.

A larga utilização de agrotóxicos na agropecuária, entre outras aplicações, gera uma série de transtornos e modificações no ambiente, seja pela contaminação das comunidades de seres vivos que o compõem, seja pela acumulação desses produtos nos segmentos bióticos e abióticos dos ecossistemas.

\section{RESULTADOS E DISCUSSÕES}

A exposição humana a agrotóxicos é um importante problema de saúde pública, pois causa diversas doenças e agravos. Com base de estudos e pesquisas vermos que o Rio grande do norte há uma projeção muito grande do fluido da utilização de agrotóxico onde em decadências disso varias questão ambientais e indisciplinares ainda ocorrem com esses avanços que é decadente ao fluido da utilização do produto

4ạ Semana de Química - IFRN, 2016 
Por isso temos que retomar e exigir um melhoramento para essa questão para que em futuras gerações não se retome ao que o estado e municípios estão se passando com problemas ambientais e socialmente.

\section{CONCLUSÃO}

Os resultados encontrados evidenciam a necessidade de serem desenvolvidas políticas de segurança alimentar baseadas na qualidade do alimento e na saúde dos trabalhadores rurais.

No Brasil, o uso de praguicidas, não sofreu acompanhamento de programas destinados à educação, conscientização e qualificação da mão-de-obra que utiliza este produto. Assim sendo, os trabalhadores ficam expostos a uma série de riscos devido ao contato direto com os produtos que se agravam com o passar do tempo (MOREIRA et al.,2002).

Um dos maiores problemas em se tratando desses métodos de conscientização, seria uma política de investimentos por parte dos órgãos governamentais. Que para montar e executar esses projetos em defesa da vida teria que se envolver com gastos e até mesmo mexer com empresas que fornecem esses produtos (agrotóxicos), que quando utilizados de forma incorreta, acabam colocando a saúde da população em risco.

Isso mostra a gravidade da situação em termos de conhecimento, que se encontra a sociedade nos dias de hoje. Quando não há órgãos reguladores e fiscalizadores que apliquem essas legislações de proteção, não será possível gerenciar uma sociedade mais voltada para sua realidade. Contudo temos ótimas leis que mereciam ser cumpridas.

O tema ainda precisa de estudos e aplicações de metodologias de Educação Ambiental visando conter o processo de banalização do uso dos agrotóxicos, insumo este com significante potencial poluidor e destrutivo, quando mal utilizado.

\section{AGRADECIMENTOS}

Os autores agradecem ao Instituto Federal de Educação, Ciência e Tecnologia do Rio Grande do Norte - IFRN, Campus Currais Novos.

A Deus pelo dom da vida, pela fé e perseverança para vencer os obstáculos. Aos meus pais, pela orientação, dedicação e incentivo nessa fase do curso e durante toda minha vida. Enfim, sou grato a todos que contribuíram de forma direta ou indireta para realização deste trabalho.

4a Semana de Química - IFRN, 2016 


\section{REFERÊNCIAS}

BEDOR, C. N. G. Estudo do potencial carcinogênico dos agrotóxicos empregados na fruticultura e sua implicação para a vigilância da saúde. 2008. 115 f. Tese (Doutorado em Saúde Pública) - Centro de Pesquisa Aggeu Magalhães, Recife, 2008. Disponível em: <http://www.arca.fiocruz.br/bitstream/icict/ 3907/2/000014.pdf>. Acesso em: 01 agos. 2016

CONSELHO NACIONAL DE SEGURANÇA ALIMENTAR E NUTRICIONAL. A Segurança Alimentar e Nutricional e o Direito Humano à Alimentação Adequada no Brasil: indicadores e monitoramento da constituição de 1988 aos dias atuais. Brasília, DF. Novembro de 2010. 284 páginas.

PERES, F.; MOREIRA, J. C. Saúde e ambiente em sua relação com o consumo de agrotóxicos em um pólo agrícola do Estado do Rio de Janeiro. BrasilCad. Saúde Pública, Rio de Janeiro, 23 Sup 4:S612-S621, 2007. Disponível em: <http://www.scielo.br/pdf/csp/v23s4/13.pdf> . Acesso em: 05 agos. 2016.

RANGEL, C. F.; ROSA, A. C. S.; SARCINELLI, P. N. Uso de agrotóxicos e suas implicações na exposição ocupacional e contaminação ambiental. Cad. Saúde Colet., Rio de Janeiro, 19 (4): 435-42-435. 2011.

Disponível

em: <http://www.iesc.ufrj.br/cadernos/images/csc/2011_4/artigos/csc_v19n4_435442.pdf> . Acesso em: 02 agos. 2016.

RODRIGUES, W. COSTA. Metodologia Científica. FAETEC/IST, Paracambi, 2007. Disponível em: <http://professor.ucg.br/sitedocente/admin/arquivosupload/ 3922/material/willian costa rodrigues_metodologia_cientifica.pdf>. Acesso em: 01 agos. 2016.

SOARES, W. L. Uso dos agrotóxicos e seus impactos à saúde e ao ambiente: uma avaliação integrada entre a economia, a saúde pública, a ecologia e a agricultura. 2010. 150 f. Tese (Doutorado em Saúde Pública) - Escola Nacional de Saúde Pública Sergio Arouca, Rio de Janeiro, 2010.2 Disponível em: 
<http://bvssp.icict.fiocruz.br/pdf/25520_tese_wagner_25_03.pdf>. Acesso em: 05 junh. 2016.

TEIXEIRA, Jules Ramon Brito et al. Intoxicações por agrotóxicos de uso agrícola em estados do Nordeste brasileiro, 1999-2009. Disponível em <http://www.scielosp.org/pdf/ress/v23n3/1679-4974-ress-23-03-00497.pdf>. 